Litinfinite Journal

ISSN: 2582-0400 [Online]

CODEN: LITIBR

Vol-1, Issue-1 (2 $\left.2^{\text {nd }} J u l y, 2019\right)$

Page No: 70-77

DOI: 10.47365/litinfinite.1.1.2019.70-77

Section: Article

\title{
One Hundred Years of Solitude: Reading Magic Realism and Alienation in their different aspects
}

\author{
Samaresh Mondal \\ Doctoral Research Scholar \\ Indian Comparative Literature Department, Assam University (Central) \\ Mail i.d.-mondalsamaresh199@gmail.com
}

\begin{abstract}
Latin American Nobel laureate novelist Gabriel Garcia Marquez's groundbreaking novel One Hundred Years of Solitude was published in 1986. The reason for choosing this novel is that it is hugely popular and admired not only in Latin America but in the whole world literature. The novel changes the author's life and draws the world's attention to Latin American literary lessons. In fact, Marquez spent much of his childhood and adolescence in the village of Arakataka. In that village full of poverty, various superstitions he weaves this story of magic and realism. Happiness and sorrow in his daily life is a world surrounded by wonder, a world that is in front of everyone but it does not catch everyone's eye - it flies away in the blink of an eye. The story of Makondo village was later created by accumulating these various experiences. This paper discusses alienation and Postmodern thinking, clubbing it with Magic Realism.
\end{abstract}

Keyword: Magic Realism, One Hundred Years of Solitude, Postmodern Novel, Postmodernism, Comparative Literature

\section{নিঃসঙ্গতার একশ বছ্র: ডাদুবাস্তবতা ও নিঃসঙ্গতীবনের ভিন্ন সংরূপ}

সারসংক্ষেপ

লাতিন আমেরিকার নোবেল বিজয়ী ঔপন্যাসিক গ্যাব্রিয়েল গার্সিয়া মার্কেসের ১৯৬৭ সালে প্রকাশিত যুগান্তকারী উপন্যাস ‘নিঃসঙ্গতার একশ বছর। এই উপন্যাসটি নির্বাচনের কারন শুধু লাতিন আমেরিকায় নয় গোটা বিশ্বসাহিত্যে বিশাল জনপ্রিয় ও বহুসমাদৃত। উপন্যাসটি লেথকের জীবন বদলে দেয় আর বিশ্ববাসীকে লাতিন আমেরিকান সাহিত্য পাঠে মনোযোগী করে তোলে।

আসলে, মার্কেসের শৈশব ও কৈশোরের অনেকটা সময় কেটেছে আরাকাতাকা গ্রামে। যে গ্রামটিতে পরিপূর্ণ দারিদ্রিক্লিষ্ট,নানারকস কুসংস্কার। তাঁর দৈনন্দিন জীবনযাত্রায় সুথ দুঃথ জীবন নিয়ে বিষয়ে ঘেরা জগৎ,যে জগৎ সবার সামনে আছে কিন্তু সবার চোথে ধরা দেয় না - চোথের পলকে উড়ে যায়। এইসব নানান 


\section{Litinfinite Journal}

ISSN: 2582-0400 [Online]

CODEN: LITIBR

Vol-1, Issue-1 (2 $\left.2^{\text {nd }} J u l y, 2019\right)$

Page No: $70-77$

DOI: 10.47365/litinfinite.1.1.2019.70-77

Section: Article

অভিজ্ঞতাকে সঞ্চয় করে গরবর্তীতে সৃষ্টি হয়েছিল মাকোন্দো গ্রামের বিবরণ। আর এই গ্রামকে ঘিরে রচিত হয়েছিল মার্কেসের বিখ্যাত উপন্যাস “নিঃসঙ্গতার একশ বছর” (Cienaños de soledad) গার্সিয়া মার্কেস নিজেই বলেছেন, মাকোন্দো একটা কাল্পনিক জগৎ। বাস্তেবের সঙ্গে কোনো অস্তিত্ব নেই। তিনি তাঁর আম্মজীবনীতে সুন্দরভাবে তুলে ধরেছেন কীভাবে মাকোন্দো নামটা মাথায় আসে। যেথানে তিনি শৈশব ও কৈশোর জীবন অতিবাহিত করেছেন। আরাকাতাকা গ্রামের বাড়ি বিক্রির জন্য মায়ের সঙ্গে আসে একেবারে আদ্যিকালের ট্রেনে চড়ে। ট্রেনে করে আসা মা ও ছেলের কথোপকথন বর্ণনা দিয়েছেন তিনি। মার্কেস লক্ষ্য করলেন, ট্রেনে করে যে সব যাত্রীরা আসছিল, তারা নিজ নিজ গন্তু্্যে নেমে যায় এবং তিনি ও তাঁর মা ছাড়া গোটা ট্রেন থালি হয়ে যায়। ট্রেনে আসতে আসতে জানালা দিয়ে মার্কেস দেথে, তাঁর ছোটবেলার চেনা পরিসর সময়ের সঙ্গে কতটা বদলে গেছে। মা 3 ছেলে দুজনেই এতটাই নিঃসঙ্গ হয়ে গেছে যে, নিজেদের কথা অগরজনের কাছে ব্যক্ত করতে পারছে না। বর্তমান সন্দর্ভে আমি তুলনামূলক সাহিত্যের দৃষ্টিকোনের উপরেই গুরুত্ব আরোপ করছি।

সূচক শব্দ: নিঃসঙ্গ, বাস্তবতা, ভাবনাচিন্তা, কল্পনা, দুঃথ, কথোপকথন, ভৌগোলিক

“যথন অপ্রত্যাশিত বাস্তবতার পরিবর্তন (অলৌকিক) থেকে উঠে আসে তথন চমৎ কারনিভ্ভুলভাবেচমৎ কার হতে শুরু করে’।

----আলেহোকার্থেন্তিয়র (১৯8৯)

উপন্যাসে গ্যাব্রিয়েল গার্সিয়া মার্কেস ‘মাকোন্দো' নামক কাল্পনিক স্থানের পটভূমিতে ঐশ্বর্শময় অদ্ভূত এক জগৎ নির্মান করেছেন। পাঠক মাত্রেই ভাবতে পারেন ‘মাকোন্দো' শিল্পন্নত দেশ, তা নয়। এটি তৃতীয় বিশ্বের একটি দেশ। কল্পনা-রূগকথা সদৃশ্য জগতে এমন সব বিস্ময়কর ঘটনা ঘটে যা আমাদের অভিজ্ঞতার বাইরে কিন্তু পাঠক হিসেবে আমরা যেটুকু ভাবতে পারি তা দেশ ও সমাডে উদ্বেগের বিযয় হয়ে দাঁড়ায়। আজ প্রায় অর্ধশতাব্দী তথা একান্ন বছ্যর অতিক্রান্ত হয়ে যাবার পরেও বাংলা পত্র পত্রিকা কিংবা বাংলায় অনুবাদ প্রকাশ দেথি তা বোঝা যায়। উগন্যাসটি পড়তে পড়তে আমাদের মনে হয় আমরা ‘মাকোন্দো’-র দেশেই বাস করছি। আজ স্বাধীনতার বাহাত্তর বছুর অতিক্ঞান্ত করেছি। আমরা ‘মাকোন্দো’-র নয়া ঔপনিবেশিক চাকচিক্য দেথি শুধুমাত্র বড় বড় শহরে, ভারতেও, বাকি দেশ গ্রামীণ ভারতে অবাধ শোযণ লুন্ঠন - ধর্ম ব্যবসা চলে। বিদেশি প্রভুর জায়গায় দেশিরা আসলেও তাদের বিলাস বহুলতা এবং ফ্ষমতার অগব্যবহার কমার কোনো কীী সম্ভাবনা নেই। শহর তথা দেশে বৈষম্য বেড়েছে, গ্রামীণ সংস্কৃতির যা অবশিষ্ট আছে তা কুসংস্কার এবং মধ্যযুগীয় আচার-বিচারে বিগন্ন। পত্তনের সময় ‘মাকোন্দো' ছিল যথার্থই স্বাধীন। 


\section{Litinfinite Journal}

ISSN: 2582-0400 [Online]

CODEN: LITIBR

Vol-1, Issue-1 (2 $\left.2^{\text {nd }} J u l y, 2019\right)$

Page No: $70-77$

DOI: 10.47365/litinfinite.1.1.2019.70-77

Section: Article

প্রতিষ্ঠাতা হোসে আর্কাদিও বোয়েন্দিয়া নিজে পরিশ্রমী চাষি। তাঁর নেতৃত্বে কিছু সংখ্যক মাকোন্দোবাসির আন্তরিক চেষ্টায় অন্যান্য গ্রামের থেকে এগিয়ে যায় এই গ্রাম। সময়ের সঙ্গে সঙ্গে পরিবর্তিত হচ্ছে অর্থাৎ আধুনিকতাকে ধরতে চাইছে। ‘কাদামাটি এবং ছিটেবেড়া'-র জায়গায় তৈরি হতে লাগল গাকাবাড়ি। বহিরাগতরা যথন বাড়ির নীল সাদা রং করার আদেশ দেয় তথন হোসে আর্কাদিও বোয়েন্দিয়া তীব্র প্রতিবাদ করে নিজের কাগজথানা ছিঁড়ে দেয় এবং বলে ‘এথানে কারও কোনো কর্তৃত্ব দরকার নেই'। আসলে হোসেআর্কাদিওর মাধ্যমে গ্রামটি উন্নয়নের দিকে ধাবিত হয়। সেথানে কারো কোনো মৃত্যু ঘটেনি। এমনকি ধর্মপ্রতিষ্ঠানও তৈরি হয়নি, ধর্মবিশ্বাস বলে কিছু নেই। বোয়েন্দিয়া পরিবার বহিরাগতের দিকে আঙুল তোলে। উপন্যাসের মূল কাহিনি বোয়েন্দিয়া পরিবার - যাকে কেন্দ্র করে সাত প্রজন্মের কাহিনি নির্মাণ করেন মার্কেস। আসলে প্রথম প্রজন্মে যে স্বাধীন, সরল এবং যূথবদ্ধ জীবনধারায় প্রবাহিত হয় তা দীর্ঘায়িত হয় না। মাকোন্দোয় দ্রুত পরিবর্তন घটতে থাকে। এই নামে কোনো স্থানের কথা জানা ছিল না লেখকের। প্রাসঙ্গিকভাবে কলম্বাসের আমেরিকা আবিষ্কারের কথা চলে আসে (মাকোন্দোর কাছে ঐকটি পরিত্যক্ত জাহাজ দেথা যায়)। তৎকালীন সময়ে স্পেনীয় '(কোনকিস্তাদোর’রা প্রবল প্রতাপে ‘নতুন বিশ্ব', 'নতুন ভাযা', ‘নতুন ধর্ম' চাপিয়ে দিয়ে প্রভুত্ব কায়েম করে।

এ তো গেল প্রথম জীবনের কথা। পরবর্তী প্রজন্মে শুরু হয় লোভ, লালসা, বহুগামিতা এবং অজাচার। এর ফলে জন্ম নেয় বৈধ, অবৈধ সন্তান, অবাধ যৌনাচার, এসব সত্বেও মা উরসুলা সবার প্রতি সমান যত্ন নেয়। এই সাহসী মা সংসারের রান্নাঘরের ‘ময়দা' মেপে দেওয়া থেকে শুরু করে মাকোন্দোর সমস্ত কাজে এগিয়ে যায়। সংসারের বাঁধন অটুট রাথার জন্য পরিশ্রমের কোনো গ্রটি নেই। এমনকি সন্তানদের অসুথে পাচন তৈরি করে থাইয়ে দেয়।

ঔপন্যাসিক যে গ্রামের বর্ণনা দিয়েছেন, তা ধীরে ধীরে প্রকৃতির, মাটির, জলের বর্ণনা থেকে উঠে আসে মানুযের কথা, মানুযের আখ্যান। মানুমের সুখ দুঃথ, ভলো মন্দ, পাথ পুন্যেরঠাসবুনোটে ফুটে ওঠে গার্সিয়া মার্কেসের অনবদ্য উপন্যাসের উন্মোচন। লেথক লেথেন মাকোন্দো গ্রামের বাসিন্দা বুয়েন্দিয়া পরিবারের সাতभুরুষের নানা কাহিনি - যার সময়কাল একটি শতাব্দী। আর বুয়েন্দিয়া পরিবারের উত্থান পতনের সঙ্গে জড়িয়ে থাকা মাকোন্দো গ্রামটির সূচনা - এই গ্রামটিকে কেন্দ্র করে সমস্ত আথ্যানের বুনট। প্রকৃতির নিয়মে মানুযের জন্ম-মৃত্যু ঘটে কিন্তু মাকোন্দো গ্রামটি থেকে যায় মহাকালের নিরপেক্ষ কষ্টিপাথর বা প্রবহমান সময়ের স্রোত। 


\section{Litinfinite Journal}

ISSN: 2582-0400 [Online]

CODEN: LITIBR

Vol-1, Issue-1 (2 $\left.2^{\text {nd }} J u l y, 2019\right)$

Page No: $70-77$

DOI: 10.47365/litinfinite.1.1.2019.70-77

Section: Article

বোদলেয়ারের দ্বারা প্রভাবিত কবি বুদ্ধদেব বসু, সেভাবেই মেক্সিকোর ব্যতিঞসী লেথক হুয়ান রুলফোর দ্বারা গার্সিয়ামার্কেসপ্রভাবিত হয়। এ প্রসঙ্গে বলা যেতে পারে রুলফোর বিথ্যাত উপন্যাস ‘পেদ্রোপারামা'-র কথা। এই উপন্যাসে কোমালা নামক একটি গ্রাম উপন্যাসের চরিত্র হয়ে উঠেছে। হয়ে উঠেছে অতীত এবং বর্তমানের মধ্যে টানাপোড়েন এবং মানব সভ্যতার জটিল যাত্রাপথের এক নিস্পৃহ সাষ্কী। এই উপন্যাসের মূল কাহিনি স্মৃতি রোমন্থন আর কোমালা সেখানে অনেক মানুমের অজস্র স্মৃতির এক ক্যালাইডোস্কোপ। স্মৃতির তাড়নায় ব্যঞন্যা পাল্টে যায়। মানুফের সুস্থ স্বাভাবিক জীবনকে ধ্নংস করে মানুযের আধিপত্য ও আকাখ্যা। ঠিক সেভাবেই কোমালার চমৎকার অতীত ধ্বংসস্তুপে পরিনত হয় নিষ্ঠুর বর্তমানের অভিমাতের স্বারা। উপন্যাসের শেমে দেথা যায় কোমালা আসলে এক মৃত জনগদ, মৃতদের গ্রাম।

উপন্যাসে মাকোন্দোর নির্মাণ অনেকটাই সেরমই। কাল্পনিক অদ্ভূদ নামক গ্রামটির (মালোন্দো) সঙ্গে মিশে রয়েছে স্বগ্নে ও বাস্তেবে মোড়া গার্সিয়া মার্কেসের শৈশবের অকপট ঘটনা এবং লাতিন আমেরিকার মানুমের দুঃথ-বেদনা-হতাশ-নকশার সূফ্ষ্ বুনন। মানুমের অস্তিত্ব সংকটের কাহিনি এবং সেই সংকটকাটিয়ে ওঠার প্রয়াস এবং ব্যর্থতা। সেই ব্যর্থতার মধ্যে পরিস্ফূট হয়ে ধরা পড়েছে আধুনিক সভ্যতার আসল সংকট, निঃসঙ্গতা।

মার্কেস আরাকাতায় গ্রাম্য পরিবেশে যৌথ পরিবারের মধ্যে ছেলেবেলা অতিবাহিতকরেছে। তাঁর ঠাকুমা মূর্থ, তাঁর কাছ থেকে কথাশিল্পী হওয়ার প্রথম পাঠ নেন। যে গ্রামটির সঙ্গে জড়িয়ে রয়েছে বাস্তুব অবাস্তেরের সংসিশ্রণ। যে পরিবেশ, পরিস্থিতির মধ্যে তিনি বড় হয়েছেন সেই পরিবেশের ছায়া ফেলেছে শুধু ‘ নিঃসঙ্গতার শতবর্ষ' নয়। মার্কেসের লেথা প্রায় সব উপন্যাস এবং ছোটগল্পে যা পরবর্তী দিনে সংজ্ঞায়িত হয়েছে জাদুবাস্তবতা বলে।

‘জাদু' এবং ‘বাস্তুবতা’ শব্দ দুটি আলাদা। জাদুর সঙ্গে বাস্তুবতার বিস্তুর ফারাক। এই দুটি বিষয় এক হয়ে সাহিত্যে নতুন আবেদন সৃষ্টি করেছে। ইংরেজিতে এটি ম্যাডিকরিয়ালিডম নামে পরিচিত। সাহিত্যে এটি এমন এক সুপরিচিত নাম একটাকে ছাড়া অন্যটা চলে না।এই সম্পর্কে বিশিষ্ট মেক্সিকান সাহিত্য সমালোচক লুই লীল বলেছেন, আभনি यদি ব্যাথ্যা করতে পারেন এটি কি, তাহলে তা জাদুবাস্তবতাই নয়। অর্থাৎ জাদু বাসুবততা এমন এক বিষয় যা ব্যাথ্যা করা সহত নয়। বর্তমান বিশ্বসাহিত্যে জাদুবাস্তবততা এক উগ্ত্বল সত্য। অত্যধিক জনগ্রিয় এবং প্রচুর আলোচিত উপাদান। ব্যপারটি সম্বন্ধে আমার মনে হয়েছে, লেথায় উপস্থাপিত ঘটনাটি घটবে জাদুর মতো, মুহুর্তের মধ্যে। কিন্তু সচেতন পাঠক মাত্রই অনুভব করবেন, তাতে এমন কিছু পাবেন, যা 


\section{Litinfinite Journal}

ISSN: 2582-0400 [Online]

CODEN: LITIBR

Vol-1, Issue-1 (2 $\left.2^{\text {nd }} J u l y, 2019\right)$

Page No: $70-77$

DOI: 10.47365/litinfinite.1.1.2019.70-77

Section: Article

থেকে বোঝা যাবে তার মূলে একটি গভীর সত্য আছে। যেখানে কোনো প্রশ্নের অবকাশ নেই বরং এটি একটি চরম বাস্তুবতা।

\section{জাদুবাস্তবতা উৎপত্তির সূত্রभाত কোথায়?}

এটি থেয়াল করলে দেথা যাবে, মধ্যयুগে রচিত মঙ্গলকাব্যের দিকে। চন্ডীমঙ্গলে উল্লেথিত ‘কালকেতু উপাথ্যানে’ এর সন্ধান পাওয়া যায়। কেননা দেবী চন্ডী একেক সময় একেক রূপ ধারণ করেছেন। হতে পারে জাদুবাস্তবতারই রূপান্তর। এছাড়াও আরব্য রজনীর কাহিনি তে জাদুবাস্তবতার সন্ধান মেলে- ‘ম্যাজিক রিয়ালিজম' কথাটি প্রথম ব্যবহার করেন একজন জার্মান শিল্প সমালোচক ফ্রাঞরোহ ১৯২৫ সালে।তিনি ম্যাডিক রিয়ালিজমের আধুনিক সংজ্ঞা দিয়েছেন, বিশ শতকের মাঝামাঝি সময়ের বিথ্যাত ম্যাজিকরিয়ালিজমের লেথক আলেহোকার্থেন্তিয়র। মার্কেসের জনগ্রিয়তার অন্যতম কারন বলা যেতে পারে ম্যাডিকরিয়ালিজম বা জাদু বাস্তুবতা। দক্ষিণ আমেরিকার সাহিত্যে বেশ সাধারন একটি বিষয় ম্যাডিকরিয়ালিজম। মার্কেসের লেথার মধ্যে সারা পৃথিবীতে এটি আরো বেশি করে ছড়িয়ে পড়ে।

উপন্যাসটির তেবোয়েন্দিয়া পরিবার এবং মাকোন্দোর গল্প তো আছে এছাড়াও কাহিনি রয়েছে প্রকৃতি এবং মানুযের নিবিড় মিশে থাকার। প্রকৃতি থেকে মানুযের বিষ্ছিন্নতা এবং উত্থান পতনের মধ্যে দিয়ে নেমে আসে নিঃসঙ্গতা এবং ধ্নংসের কাহিনি। মাকোন্দোর মাটিতে বোয়েন্দিয়া পরিবার বসবাস শুরুর সময় থেকে আপাত সারল্যের মধ্যে নিহিত ছিল অবক্ষয় আর ধ্বংসের বীজ। ধ্বংস আর অবক্ষয়ের বীজ বপন শুরু হয় হোসে আর্কাদিও বোয়েন্দিয়া এবং উরসুলার বিয়ের মাধ্যমে আসলে এদের সম্পর্ক হচ্ছে থুডুতুতো ভাইবোন, অবৈধ সম্পর্কের পরিণতি। তাদের কাহিনি অতীতের দিকে তাকালে দেথা যায় মাকোন্দোতে বসবাস শুরুর আগে ওদের বাস ছিল অনাম, অথ্যাত গ্রামে। কৈশোর থেকে তারা একে অপরের প্রতি অনুরক্ত ছিল। সময়ের সঙ্গে সঙ্গে যথন পরিস্থিতি বদলাতে শুরু করে তারা বিয়ে করে,সহবাস করে কারন অজাচারজনিত পাপের ভয়ে। গ্রামের মানুষদের বিশ্বাস ছিল যে, ভাইবোনের সম্পর্কে বিয়ে হলে সুস্থ ও স্বাভাবিক সন্তান জন্ম হয় না। এইসব ভাবনাচিন্তা জ্ঞাত থাকার গরেও শেশে তারা বিয়ে করে। সুস্থ ও স্বাভাবিক তো নয়ই বরং পেছনে শুয়োরের লেজ যুক্ত সদ্যোজাত সন্তানের জন্ম দেয়। (হাবিব, পৃঃ৩৪৯)

এইভাবেই শুরু হয় বোয়েন্দিয়া পরিবারে পাপের বীজ বগন। অপর দিকে প্রুদেন্সিওর আহ্মা বিবেকযন্তণা হয়ে ঘুরে বড়ায়, ওই পরিবার থেকে মুক্তি পাবার জন্য। তারা মুক্তির সন্ধানে নতুন বাসভূমির থোঁজে পাড়ি দেয়। 


\section{Litinfinite Journal}

ISSN: 2582-0400 [Online]

CODEN: LITIBR

Vol-1, Issue-1 (2 $\left.2^{\text {nd }} J u l y, 2019\right)$

Page No: $70-77$

DOI: 10.47365/litinfinite.1.1.2019.70-77

Section: Article

প্রাচীন শহর রিও আচার সন্ধানে বেরোয় তারা। ভাগ্যান্বেষী মানুযেরা প্রকৃতির সঙ্গে দীর্ঘ লড়াই করে যথন তারা ক্লান্ত তথন মাকোন্দোতে এসে (ৌঁঁছায়। আর সেথানেই গড়ে ওঠে তাদের নয়া বাসভূমি।

মাকোন্দো গ্রাম যথন তৈরি হয়, তথন তা ছিল প্রকৃতির আয়না। উপন্যাসে লক্ষ্যণীয়, “অনেক বছুর পর যথন কর্নেল আউরোলিয়ানো বুয়েন্দিয়া ফায়ারিংস্কোয়াডের সামনে এসে দাঁড়ায়, তথন হঠাৎ তাঁর মনকে বিদ্ধ করে স্মৃতির ব্যাথা। সেই অনেকদিন আগের এক বিকেলের স্মৃতি, যেদিন তার বাবা হাত ধরে বরফ দেথাতে নিয়ে গেয়েছিল। মাকোন্দো তথন নেহাতই এক গন্ডগ্রাম, যেখানে কাদামাটি আর নলখাগড়া দিয়ে বানানো গোটা কুড়ি বাড়ি। তাদের পাশ দিয়ে শান্ত ধারায় কলকল করে বয়ে চলেছে এক স্বচ্ছুলের নদী। জলের নিচে প্রাগৈতিহাসিক ডিমের মতো ছড়িয়ে রয়েছে বিশাল বিশাল পাথর। এতো মসৃন যেন গালিশ করা। পৃথিবীটা তথন একেবারে সদ্যোজাত। তার বেশিরভাগ জিনিসের তথনও নাম দেওয়া হয়নি। আঙুল দিয়ে চিনিয়ে দিতে হয়"। ( হাবিব, পৃ:২৮৫)।

বুয়েন্দিয়া পরিবারে কাম ও ক্রোধের মত্য দিয়ে যে পাপের সূত্রপাত, সেই পাপের চাকা এগিয়ে যায় নানা কুবৃত্তিকে আশ্রয় করে। তার মধ্য দিয়ে সবচেয়ে বড় শঙ্রু হিসেবে প্রবেশ করে লোভ। এরইমধ্যে কোথা থেকে হাজির হয় যাযাবর জিগসিসেলকিয়াদেস, যার আসাধারণ জাদু ফ্কমতার দ্বারা বশ করে ফেলে হোসে আর্কাদিও বুয়েন্দিয়াকে। জাদুর প্রভাবে গৃহগালিত পশুর বিনিময়ে কিনে ফেলে একটা চুম্বক এবং ধনী হওয়ার প্রত্যাশা নিয়ে মাকোন্দোর মাটিতে সোনা থুঁচতে শুরু করে হোসেআর্কাদিও। এরই লোভে আর্কাদিও বিষ্দিন্ন হতে শুরু করে। শুরু হয় শতবর্শব্যাপী একাকিত্বের উপাথ্যান, নিঃসঙ্গজীবন। আশ্চর্যের একটি বিষয় তা হল, গ্রাজ্ঞ মেলকিয়াদেস হোসে আকার্দিওকে একবারের জন্য হলেও ঠকাবার চেষ্টা করেনি। মেলকিয়াদেস জাদুর প্রভাবে এমনভাবে বশ করেনিয়েছ্ছিল যে, আর্কাদিও নেশায় মত্ত থেকে একের পর এক ‘উদ্টট থেলনা’ জিনিস কিনতে থাকে। আর দাম মেটাতে থাকে উরসুলার সयন্ন সঞ্চয় থেকে, তা গৃহ্ালিত পশুই হোক বা বহুমূল্য স্বর্ণমুদ্রাই হোক। সে সমস্ত পরীক্যা নিরীক্ষায় ব্যর্থ হয়েও হার মানে না। তাকে তাড়া করে বেড়ায় ধনী হওয়ার স্বপ্ন। সে এতটাই ধনী হওয়ার স্বগ্লের উন্মাদনায় মেতেছে যে, এমনকি পরিবারের সঙ্গে সম্পর্ক ছিন্ন হয়। যে গ্রামের মাটিতে পেশির জোরে সোনা ফলাতে পারতো, সে মাটিতে পাগলের মতো সোনা থোঁজার চেষ্টা করে। অথচ তারই উদ্যোগে এবং ভাবনা চিন্তায় মাকোন্দো বহির্বিশ্বের থেকে বিষ্ছিন্ন থেকেও পরিণত হয়েছিল সবচেয়ে সুসংগঠিত, সুথী ও কর্মব্যস্ত গ্রামে।যে গ্রামে প্রতিটি বাসিন্দার বয়স তিরিশের কম এবং যে গ্রাম কথনো মৃত্যু দেথেনি। বুয়েন্দিয়া পরিবারকেই ওই গ্রামের আদর্শ পরিবার বলে গণ্য করা হতো । মাকোন্দোতেবুয়েন্দিয়া বাড়ির স্টাইলে ছিল আরো অন্যান্য সব বাড়ি। 


\section{Litinfinite Journal}

ISSN: 2582-0400 [Online]

CODEN: LITIBR

Vol-1, Issue-1 (2 $\left.2^{\text {nd }} J u l y, 2019\right)$

Page No: $70-77$

DOI: 10.47365/litinfinite.1.1.2019.70-77

Section: Article

সময়ের সঙ্গে সঙ্গে বদলে যায় অঞ্চলটির আর্থসামাডিক প্রেক্কাপট। তুলনামূলক সাহিত্যের ইতিহাসবিদ্যার (Historiography) পদ্ধতি অবলম্বনে দেথা যায় যে, গোটা প্রক্রিয়াটাই ইতিহাসের প্রেক্ষাপটে তৈরি। মা উরসুলার আবিস্কার করা জলাভূমি পেরোনোর পথ দিয়ে যোগাযোগের সূত্রপাত ঘটে বাইরের জগতের সামনে। কৃষিভীবী থেকে শুরু করে নানানধরণের ব্যবসায়ী আসে গ্রামটিতে। গ্রাম সমৃদ্ধ হতে থাকে। আসে সরকার, আসে পাদ্রিরা, আসে পুলিশ। গ্রামটির আমূল পরিবর্তন ঘটতে থাকে অর্থাৎ আধুনিকতাকে ধরতে চাইছে। এর ফলে মাকোন্দো আর আগের মতো নেই। কাদামাটি আর ছিটেবেড়ার, পরিবর্তে আসে ‘ইটের ঘর, ইঁটের দেয়াল, কাঠের জানালা, সিমেন্টেরমেঝে......' সেই সঙ্গে আসে অনাচার, অজাচার, প্রেমহীন শরীরী উন্মাদনায় ঝাঁপ দেয় বুয়েন্দিয়া পরিবারের প্রজন্মের পর প্রজন্ম। সব সন্পর্কের উত্তাপ ছাপিয়ে গিয়ে শুষু পড়ে থাকে নিঃসঙ্গতা।

মার্কেস তাঁর আম্মজীবনীতে বলেছেন, আরাকাতাকে ঘিরে অতীতের যে শৈশবের স্মৃতি ছিল ট্রেনে মায়ের সঙ্গে যেতে যেতে লক্ষ্য করলেন শহরটি নিঃসঙ্গ নিঝুম ধরা রূপ। লেথকের মনে হয়েছিল ওই শহরের গথে চেনা মানুষকেও আর চেনা যায় না। গোটা শহরটা একটা অজানা ঘোরের মধ্যে আবর্তিত। সর্বত্র ছড়িয়ে রয়েছে বিযন্নতা এবং নিঃসঙ্গতা। আর এসব কারনেই মাকোন্দোতে আষ্টেপৃষ্টেরয়েছে স্বগ্ন ও বাস্তুেে ঘেরা ব্যক্তিগত স্মৃতি বিস্মৃতি এবং লাতিন আমেরিকার মানুষের দুঃথ বেদনা-হতাশা-মোড়া অস্তিত্বের সংকট। লাতিন আমেরিকা পরিচয়টাই তো সাম্রাজ্যবাদের দেগে দেওয়া, সেথানে মানুয হারিয়ে ফেলেছে তার অস্তিত্বের বিশুদ্ধতা, তার ভাযা, ধর্ম, সংস্কৃতি এবং প্রাক-স্প্যানিশ যুগের সমস্ত অভিজ্ঞান। তার পূর্বপুরুমের রক্তে মিশে গেছে বিদেশি শাসকদের রক্ত। বিজেতাদের ভাযা ছাড়া তার কথা বলার উপায় নেই। তীব্র যন্ত্রণায় দগ্ধ হতে হতে অতীতকে থুঁজে বেড়ায়, স্বপ্নকে তাড়া করে ফেরে উদ্টট সব কল্পকাহিনি - গ্রাম থেকে জনপদ, জনপদ থেকে নগর এবং নগর থেকে ধ্বংসস্তূপের অনিবার্য যাত্রাপথ। जিপসিদের হাত ধরেই মাকোন্দোতে অলৌকিকের প্রবেশ। জিপসি মেলকিয়াদেস জাদুবিদ্যায় এতটাই পারদর্শী যে, তার চুম্বকের টানে রান্নাঘর থেকে হুড় মুড় করে বেরিয়ে আসে লোহার তৈরি বাসনগত্র। মেলকিয়াদেসের হাত ধরেই জাদুবিদ্যার প্রভাব ছড়িয়ে পড়ে গোটা মাকোন্দো জুড়ে।

মাকোন্দোতে যে ঘটনাগুলো ঘটেছে সেগুলিকে কেন্দ্র করে তৈরি হয় রূগকথা বা মিথ। মিথের আগমন বিভিন্ন সূত্র ধরে। যেমন 'মাকোন্দো' কথাটির উৎস সম্ভবত উত্তর কঙ্গোর ভামায় কলার বহুবচন ‘মানকোন্দা'। বিভিন্ন ধরণের ব্যবসায়ীরা মাকোন্দোতে এসে কলা চাষ, আফ্রিকা থেকে ঐীতদাস হিসেবে নিয়ে আসা কৃষি শ্রমিকদের বংশধর, কলার গন্ধ এবং কলা চাষ বন্ধ হয়ে গেলে মার্কিন কোম্পানির ফেলে যাওয়া কলার বর্জ্য মিলেমিশে এক ধরনের মিথ হয়ে ওঠঠ। এক সময় যেটা বাস্তব ঘটনা ছিল, তারই গায়ে জলহাওয়া লেগে তা 


\section{Litinfinite Journal}

ISSN: 2582-0400 [Online]

CODEN: LITIBR

Vol-1, Issue-1 ( $2^{\text {nd }}$ July, 2019)

Page No: 70-77

DOI: 10.47365/litinfinite.1.1.2019.70-77

Section: Article

পরাবাস্তুব হয়ে ওঠে। যেমন, কুড়ি বচুরের যুদ্ধকে ঘিরে নানা অতিরঞ্জিত ঘটনা তৈরি হয়। এমনকি এটাও শোনা যায় যে, কর্নেল আউরেলিয়ানো বুয়েন্দিয়াকে নরখাদকরা থেয়ে ফেলেছে। যেমন শুয়োরের লেজ নিয়ে বাষ্ঠর জন্ম এবং অচিরেই সেই শিশুর পিঁপড়েরথাদ্যতেপরিনত হওয়া, আগুন ছাড়া জল ফোটা, গর্ভস্থ শিশুর কান্না পাওয়া-এইসব নানান রকমের রূপক ও মিথকে ঘিরেই তৈরি হয় মাকোন্দোর যাত্রাপথ।

শতবর্মের নিঃসঙ্গতা আসলে মাকোন্দোরই। তার বাসিন্দারা এক একটি রক্ত মাংসের গড়া মানুষ। মৃত্যু হয়েও তারা আडীবন নিঃসঙ্গতার অবসান ঘটায়। সচেতন পাঠক মাত্রেই ভাবেন, যুগ পরিবর্তনের ক্ষেত্রেও মাকোন্দো নিঃসঙ্গতার সাফীকী বহন করে। যে নিঃসঙ্গতা থেকে তৈরি হয় মাকোন্দোর একাকী যন্ত্রণার বিম্বিত, মানুমের ভালোমন্দতে গড়া এক মহাকাব্যিক আলেথ্য। নিঃসঙ্গতা মার্কেসের অন্যান্য গ্রন্থে ছড়়িয়ে পড়ে। বিশেষ করে ‘নিঃসঙ্গতা’ (Solitude) শব্দটি লাতিন আমেরিকা মানুযেরা শুনলে আজও বিভ্রান্তিতে পড়ে যায়।

Works cited

i. Bhattacharya, Buddhadev (translation). Hidden in Chile. Kolkata: Dejpab Publishing, 1995.

ii. Goswami, Vijaya. Comparative Linguistics and Sanskrit Language, Calcutta: Sanskrit Bookstore, 2009.

iii. Habib, G. H (translation). One Hundred Years of Loneliness. Dhaka: Batighar Publications, 2018.

iv. Mukherjee, Manabendra (translation). Collection of essays - Alehocarpentier. Kolkata: Dejpab Publishing, 1997.

সহায়কগ্রন্থপঞ্জি (Works cited in Bengali)

গোস্বামী , বিজয়া। তুলনামূলক ভাষাতত্ত্বও সংস্কৃত ভাষ। কলকাতা : সংস্কৃত পুস্তকভান্ডার , ১৪১৬। মুদ্রণ।

মুখোপাধ্যায় , মানবেন্দ্র ( অনুবাদ )। রচনা সংগ্রহ - আলেহোকার্পেল্ভিয়র। কলকাতা : দে' জপাবলিশিং , ১৯৯৭। মুদ্রণ।

হাবিব , জি. এইচ ( অনুবাদ )। নিঃসঙ্গতার একশো বছর। ঢাকা : বাতিঘর প্রকাশন , ২০১৭ । মুদ্রণ।

ভট্টাচার্য, বুদ্ধদেব ( অনুবাদ )। চিলিতেগোপন্। কলকাতা : দে'জপাবলিশিং , ১৯৯৫। মুদ্রণ। 